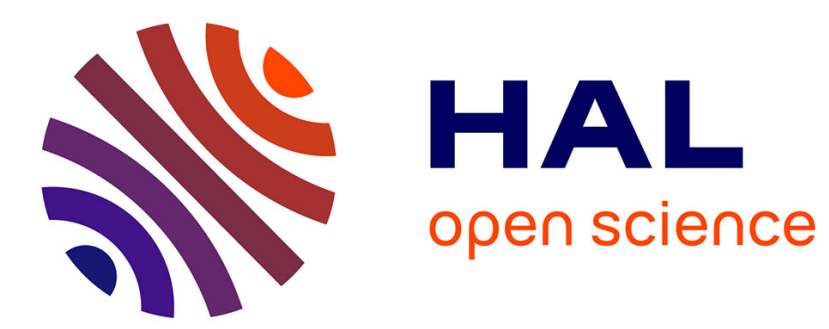

\title{
System-Level Analysis of SWIPT MIMO Cellular Networks
}

\author{
Thanh Tu Lam, Marco Di Renzo, Justin P. Coon
}

\section{To cite this version:}

Thanh Tu Lam, Marco Di Renzo, Justin P. Coon. System-Level Analysis of SWIPT MIMO Cellular Networks. IEEE Communications Letters, 2016, 10.1109/LCOMM.2016.2590424 . hal-01359555

\section{HAL Id: hal-01359555 \\ https://hal.science/hal-01359555}

Submitted on 2 Sep 2016

HAL is a multi-disciplinary open access archive for the deposit and dissemination of scientific research documents, whether they are published or not. The documents may come from teaching and research institutions in France or abroad, or from public or private research centers.
L'archive ouverte pluridisciplinaire HAL, est destinée au dépôt et à la diffusion de documents scientifiques de niveau recherche, publiés ou non, émanant des établissements d'enseignement et de recherche français ou étrangers, des laboratoires publics ou privés.

\section{(1) $\$(0)$}

Distributed under a Creative Commons Attribution - NonCommercial - ShareAlikel 4.0 


\title{
System-Level Analysis of SWIPT MIMO Cellular Networks
}

\author{
Thanh Tu Lam, Student Member, IEEE, Marco Di Renzo, Senior Member, IEEE, and \\ Justin P. Coon, Senior Member, IEEE
}

\begin{abstract}
We introduce a tractable approach for studying the feasibility of multiple-antenna cellular networks, where lowenergy devices decode information data and harvest power simultaneously. Tools from stochastic geometry are used to quantify the information rate vs. harvested power tradeoff. Our study unveils that large-scale antenna arrays and ultra-dense deployments of base stations are both necessary to harvest, with high reliability, an amount of power of the order of a milliwatt.
\end{abstract}

Index Terms-Cellular Networks, MIMO, SWIPT.

\section{INTRODUCTION}

Simultaneous Wireless Information and Power Transfer (SWIPT) is a technology where the same radio frequency signal is used for data transmission and for replenishing the battery of Low-Energy Devices (LEDs) [1]. The design of SWIPT cellular networks [2] poses new research challenges. Cellular networks are designed based on the assumption that the interference has a negative impact on Wireless Information Transfer (WIT) [3]. The same interference, on the other hand, is a natural source of power for improving Wireless Power Transfer (WPT) [4]. As a result, the development of interference management techniques that exploit interference for WPT and counteract it for WIT plays a fundamental role.

Multiple-Input-Multiple-Output (MIMO) systems constitute a promising solution to manage and exploit the interference at once [5]. At the receiver, multiple antennas enhance data reliability and increase the harvested power via spatial diversity. At the transmitter, multiple antennas improve information and power transfer via spatial beamforming. Spatial beamforming, however, results in interference isolation that reduces the harvested power. Using MIMO, thus, introduces, several trade-offs in SWIPT cellular networks, which have not been quantified yet [5]. This is the objective of this Letter.

Most works on SWIPT are focused on small-scale networks [5]. Its potential in large-scale networks is, on the other hand, less investigated. In [6], [7] and [8], [9] relay-aided networks and ad hoc networks are studied. These papers, however, consider single-antenna transmission. In [10], ad hoc networks with multiple-antenna transmitters are studied. The analysis, however, is not applicable to cellular networks. SWIPT cellular networks are investigated in [11] and [12] for legacy and millimeter-wave frequencies. In both cases, directional antennas are taken into account but MIMO is not.

Letter received March 7, 2016; revised June 5, 2016. The editor coordinating the review of this letter and approving it for publication was N. Zlatanov.

T. Tu Lam and M. Di Renzo are with the Laboratoire des Signaux et Systèmes, CNRS, CentraleSupélec, Univ Paris Sud, Université Paris-Saclay, 3 rue Joliot Curie, Plateau du Moulon, 91192 Gif-sur-Yvette, France. (e-mail: lamthanh.tu@12s.centralesupelec.fr, marco.direnzo@12s.centralesupelec.fr).

J. P. Coon is with the Department of Engineering Science, University of Oxford, Parks Road, OX1 3PJ, Oxford, UK. (e-mail: justin.coon@eng.ox.ac.uk).

This work is supported in part by the EC through the H2020-ETN5Gwireless project (grant 641985) and the EPSRC through the Spatially Embedded Networks project (grant EP/N002350/1).

Digital Object Identifier XXX.XXX/LCOMM.XXX.XXX.
We introduce a tractable approach to quantify the potential of MIMO in SWIPT cellular networks. Maximum Ratio Transmission (MRT) and Maximum Ratio Combining (MRC) at Base Stations (BSs) and LEDs are considered. The locations of the BSs are modeled as points of a Poisson Point Process (PPP) and stochastic geometry is used for system-level analysis. Three mathematical frameworks are proposed, which provide exact, approximated, and large-scale asymptotic expressions of the Joint Complementary Cumulative Distribution Function (J-CCDF) of information rate and harvested power.

Our feasibility study shows that large-scale MIMO and ultra-dense deployments of BSs are both necessary to harvest, with high reliability, an amount of power of the order of a milliwatt. In the large-scale MIMO regime, also, the J-CCDF depends only on the average strength of the intended link. ${ }^{1}$

\section{System MOdeL}

\section{A. Cellular Networks Modeling}

A downlink MIMO cellular network is considered. BSs and LEDs are equipped with $N_{t}$ and $N_{r}$ antennas, respectively. The BSs are modeled as points of a homogeneous PPP, denoted by $\Psi$, of density $\lambda$. Their transmit power is $P$. The analysis is performed for the typical LED located at the origin [3].

\section{B. SWIPT Based on Power Splitting (PS)}

The typical LED is equipped with information and energy receivers that operate according to the PS scheme [1]. The received power is split in two parts, according to the power splitting ratio $0 \leq \rho \leq 1$, and is used for Energy Harvesting (EH) and Information Decoding (ID).

\section{Channel Modeling}

The channel model accounts for Line-of-Sight (LOS) and Non-LOS (NLOS) links due to spatial blockages, as well as for path-loss and fast-fading [13]. Shadowing is implicitly accounted for via the LOS and NLOS link model [14].

1) LOS/NLOS Links: Let $r$ be the distance from a BS to the typical LED. The probability to be in LOS and NLOS as a function of $r, p_{s}(\cdot)$ for $s \in\{\mathrm{LOS}, \mathrm{NLOS}\}$, is as follows:

$$
p_{s}(r)=\left\{\begin{array}{lll}
q_{s}^{[0, D]} & \text { if } & r \in[0, D) \\
q_{s}^{[D, \infty]} & \text { if } & r \in[D,+\infty)
\end{array}\right.
$$

\footnotetext{
${ }^{1}$ Uppercase and lowercase boldface symbols denote matrices and vectors. $\mathbb{C}^{M \times N}$ is the field of $M \times N$ complex matrices. $X \sim \mathcal{C N}\left(\mu, \sigma^{2}\right)$ is a complex Gaussian Random Variable (RV) with mean $\mu$ and variance $\sigma^{2}$. $X \sim$ $\mathcal{E}(\Omega)$ is an exponential RV with mean $\Omega .(\cdot)^{*}$ is the conjugate transpose. $j=\sqrt{-1}$ is the imaginary unit. $\mathbb{E}\{\cdot\}$ is the expectation operator. $(\cdot)$ ! is the factorial operator. $\|\cdot\|_{F}$ is the Frobenius norm. $\mathbf{1}(\cdot)$ and $\mathcal{H}(\cdot)$ are indicator and Heaviside functions. $\overline{\mathcal{H}}(x)=1-\mathcal{H}(x) \cdot{ }_{p} F_{q}(\cdot ; \cdot ; \cdot), \delta(\cdot), \Gamma(\cdot)$ and $\Gamma(\cdot, \cdot)$ are generalized hypergeometric, delta, gamma and upper-incomplete gamma functions. $f_{X}(\cdot)$ and $\Phi_{X}(\cdot)$ denote the Probability Density Function (PDF) and the Characteristic Function (CF) of RV $X$.
} 


$$
\begin{aligned}
& \mathcal{J}_{v, u}^{(1)}=\int_{0}^{+\infty} \int_{0}^{+\infty} \frac{1}{\pi \omega} \operatorname{Im}\left\{\exp \left(-j \omega \frac{q_{*}}{P \mathcal{G}_{M}}\right)\left(v-\frac{j \omega}{y}\right)^{-(1+u)} \Gamma\left(1+u, \frac{\mathcal{T}_{*}}{P \mathcal{G}_{M}}(v y-j \omega)\right) \Phi_{\mathcal{I}}\left(\frac{\omega}{\mathcal{G}_{M}} \mid y\right)\right\} f_{L^{(0)}}(y) d \omega d y \\
& \mathcal{J}_{v, u}^{(2)}=\int_{0}^{+\infty} \int_{0}^{+\infty} \frac{1}{\pi \omega} \operatorname{Im}\left\{\exp \left(j \omega \frac{\sigma_{*}^{2}}{P \mathcal{G}_{M}}\right)\left(v+\frac{j \omega r_{*}}{y}\right)^{-(1+u)} \Gamma\left(1+u, \frac{\mathcal{T}_{*}}{P \mathcal{G}_{M}}\left(v y+j \omega r_{*}\right)\right) \Phi_{\mathcal{I}}\left(\frac{\omega}{\mathcal{G}_{M}} \mid y\right)\right\} f_{L^{(0)}}(y) d \omega d y
\end{aligned}
$$

where $q_{\mathrm{LOS}}^{[a, b]}+q_{\mathrm{NLOS}}^{[a, b]}=1, q_{s}^{[a, b]}$ is the probability that a link of length $r \in[a, b)$ is in state $s$, and $D$ takes into account that LOS and NLOS probabilities are different for short and long distances [14]. Assuming no spatial correlation, $\Psi$ can be split in two independent and non-homogeneous PPPs, $\Psi_{\mathrm{LOS}}$ and $\Psi_{\text {NLOS }}$, such that $\Psi=\Psi_{\text {LOS }} \cup \Psi_{\text {NLOS }}$. From (1), the density of $\Psi_{s}$ is $\lambda_{s}(r)=\lambda p_{s}(r)$ for $s \in\{\mathrm{LOS}, \mathrm{NLOS}\}$.

2) Path-Loss: The path-loss of LOS and NLOS links is $l_{s}(r)=\kappa_{0} r^{\beta_{s}}$ for $s \in\{\operatorname{LOS}, \mathrm{NLOS}\}$, where $\kappa_{0}=(4 \pi / \nu)^{2}$, $\nu$ is the wavelength and $\beta_{s}$ is the path-loss exponent.

3) Fast-Fading: All channels are assumed to be independent and identically distributed (i.i.d.) $\mathcal{C N}(0,1)$ RVs.

\section{Cell Association}

The typical LED is served by the BS providing the smallest path-loss. The other BSs act as interferers. The smallest pathloss is denoted by $L^{(0)}=\min \left\{L_{\mathrm{LOS}}^{(0)}, L_{\mathrm{NLOS}}^{(0)}\right\}$, where, for $s \in\{\mathrm{LOS}, \mathrm{NLOS}\}, L_{s}^{(0)}$ is defined as follows:

$$
L_{s}^{(0)}=\min _{n \in \Psi_{s}}\left\{l_{s}\left(r^{(n)}\right)\right\}
$$

and $r^{(n)}$ is the distance between a BS and the typical LED.

\section{MIMO CELlular NeTWORKS}

At the BSs, MRT with directive antennas is used. Twolobe antennas are considered, where $\mathcal{G}_{M}$ and $\mathcal{G}_{m}$ are the gains of main and secondary lobes, and $\omega_{M}$ is the width of the main lobe [13]. Each BS steers its main lobe towards the LED associated to it, hence the non-intended links are randomly oriented with respect to each other and uniformly distributed in $[0,2 \pi)$. Thus, the received vector, $\mathbf{y} \in \mathbb{C}^{N_{r} \times 1}$, is:

$$
\mathbf{y}=\mathbf{U}+\mathbf{I}_{\mathrm{LOS}}+\mathbf{I}_{\mathrm{NLOS}}+\mathbf{n}
$$

where $\mathbf{U} \in \mathbb{C}^{N_{r} \times 1}$ is the intended signal and $\mathbf{I}_{s} \in \mathbb{C}^{N_{r} \times 1}$ for $s \in\{$ LOS, NLOS $\}$ is the other-cell interference:

$$
\begin{aligned}
\mathbf{U} & =\sqrt{P \mathcal{G}^{(0)} / L^{(0)}} \mathbf{H}^{(0)} \mathbf{w}_{t}^{(0)} x^{(0)} \\
\mathbf{I}_{s} & =\sum_{i \in \Psi_{s}} \sqrt{P \mathcal{G}^{(i)} / l_{s}\left(r^{(i)}\right)} \mathbf{H}^{(i)} \mathbf{w}_{t}^{(i)} x^{(i)} \mathbf{1}\left(l_{s}\left(r^{(i)}\right)>L^{(0)}\right)
\end{aligned}
$$

and $\mathbf{n} \in \mathbb{C}^{N_{r} \times 1}$ is the Gaussian noise with $\mathbf{n}(\tau) \sim$ $\mathcal{C N}\left(0, \sigma_{N}^{2}\right)$ for $\tau=1,2, \ldots, N_{r}$. The indicator function in (4) is due to the cell association. The notation is as follows.

- $\mathbf{H}^{(0)} \in \mathbb{C}^{N_{r} \times N_{t}}$ and $\mathbf{H}^{(i)} \in \mathbb{C}^{N_{r} \times N_{t}}$ are the channel matrices of serving and $i$ th interfering BSs. Their elements are i.i.d., with $\mathbf{H}^{(0)}(\tau, t) \sim \mathcal{C N}(0,1)$ and $\mathbf{H}^{(i)}(\tau, t) \sim$ $\mathcal{C N}(0,1)$ for $\tau=1,2, \ldots, N_{r}$ and $t=1,2, \ldots, N_{t}$.

- $x^{(0)}$ and $x^{(i)}$ are the data symbols of serving and $i$ th interfering BSs. They are i.i.d. with zero mean and unit power, i.e., $\mathbb{E}\left\{\left|x^{(0)}\right|^{2}\right\}=\mathbb{E}\left\{\left|x^{(i)}\right|^{2}\right\}=1$.
- $\mathbf{w}_{t}^{(0)} \in \mathbb{C}^{N_{t} \times 1}$ with $\left\|\mathbf{w}_{t}^{(0)}\right\|^{2}=1$ is the beamforming vector of the serving BS. It is the eigenvector corresponding to the largest eigenvalue of $\mathbf{F}^{(0)}=\left(\mathbf{H}^{(0)}\right)^{*} \mathbf{H}^{(0)} \in$ $\mathbb{C}^{N_{t} \times N_{t}}$, which is $\chi^{(0)}=\left\|\left(\mathbf{H}^{(0)} \mathbf{w}_{t}^{(0)}\right)^{*} \mathbf{H}^{(0)} \mathbf{w}_{t}^{(0)}\right\|[15$, Eq. (33)]. Its PDF is as follows [15, Eq. (9)]:

$$
f_{\chi^{(0)}}(\xi)=\mathcal{K}_{p, q} \sum_{v=1}^{q} \sum_{u=p-q}^{(p+q-2 v) v} c_{v, u} \xi^{u} \exp (-v \xi)
$$

where $p=\max \left\{N_{t}, N_{r}\right\}, q=\min \left\{N_{t}, N_{r}\right\}, \mathcal{K}_{p, q}=$ $\left(\prod_{a=1}^{q}(q-a) !(p-a) !\right)^{-1}, c_{v, u}$ follows from [15].

- $\mathbf{w}_{t}^{(i)} \in \mathbb{C}^{N_{t} \times 1}$ with $\left\|\mathbf{w}_{t}^{(i)}\right\|^{2}=1$ is the beamforming vector of the $i$ th interfering BS. It is the eigenvector corresponding to the largest eigenvalue of $\mathbf{F}^{(i)}=$ $\left(\mathbf{G}^{(i)}\right)^{*} \mathbf{G}^{(i)} \in \mathbb{C}^{N_{t} \times N_{t}}$, where $\mathbf{G}^{(i)} \in \mathbb{C}^{N_{r} \times N_{t}}$ is the channel matrix of the LED served by the $i$ th BS.

- $\mathcal{G}^{(0)}$ and $\mathcal{G}^{(i)}$ are the directivity gains of intended and interfering links, where $\mathcal{G}^{(0)}=\mathcal{G}_{M}$ and $f_{\mathcal{G}^{(i)}}(g)=$ $\left(\omega_{M} / 2 \pi\right) \delta\left(g-\mathcal{G}_{M}\right)+\left(1-\omega_{M} / 2 \pi\right) \delta\left(g-\mathcal{G}_{m}\right)$.

At the LEDs, MRC with omnidirectional antennas is used. The demodulation vector is $\mathbf{w}_{r}^{(0)}=\mathbf{H}^{(0)} \mathbf{w}_{t}^{(0)} /\left\|\mathbf{H}^{(0)} \mathbf{w}_{t}^{(0)}\right\|$. The signals at the input of ID and EH receivers are:

$$
z_{\mathrm{ID}}=\sqrt{1-\rho}\left(\mathbf{w}_{r}^{(0)}\right)^{*} \mathbf{y}+m_{\mathrm{ID}} ; \quad z_{\mathrm{EH}}=\sqrt{\rho}\left(\mathbf{w}_{r}^{(0)}\right)^{*} \mathbf{y}+m_{\mathrm{EH}}
$$

where $m_{\mathrm{ID}} \sim \mathcal{C N}\left(0, \sigma_{\mathrm{ID}}^{2}\right)$ and $m_{\mathrm{EH}} \sim \mathcal{C N}\left(0, \sigma_{\mathrm{EH}}^{2}\right)$ are the additive noises of ID and EH receivers [11], respectively.

From (6), Shannon rate (in bits/sec), $\mathcal{R}$, and harvested power (in Watts), $\mathcal{Q}$, of ID and ED receivers are as follows:

$$
\begin{aligned}
& \mathcal{R}=B_{w} \log _{2}\left(1+\frac{P \mathcal{G}^{(0)} \chi^{(0)} / L^{(0)}}{P \mathcal{I}_{\mathrm{ID}}+\sigma_{N}^{2}+\sigma_{\mathrm{ID}}^{2} /(1-\rho)}\right) \\
& \mathcal{Q}=\rho \zeta\left(P \mathcal{G}^{(0)} \chi^{(0)} / L^{(0)}+P \mathcal{I}_{\mathrm{EH}}\right)
\end{aligned}
$$

where $B_{w}$ is the bandwidth, $0 \leq \zeta \leq 1$ is the $\mathrm{EH}$ conversion efficiency, and $\mathcal{I}=\mathcal{I}_{\mathrm{ID}}=\mathcal{I}_{\mathrm{EH}}$ is the other-cell interference:

$$
\begin{aligned}
& \mathcal{I}=\sum_{i \in \Psi_{\mathrm{LOS}}}\left(1 / l_{\mathrm{LOS}}\left(r^{(i)}\right)\right) \mathcal{G}^{(i)} \gamma^{(i)} \mathbf{1}\left(l_{\mathrm{LOS}}\left(r^{(i)}\right)>L^{(0)}\right) \\
& +\sum_{i \in \Psi_{\mathrm{NLOS}}}\left(1 / l_{\mathrm{NLOS}}\left(r^{(i)}\right)\right) \mathcal{G}^{(i)} \gamma^{(i)} \mathbf{1}\left(l_{\mathrm{NLOS}}\left(r^{(i)}\right)>L^{(0)}\right)
\end{aligned}
$$

where $\gamma^{(i)}=\left\|\left(\mathbf{H}^{(0)} \mathbf{w}_{t}^{(0)}\right)^{*} \mathbf{H}^{(i)} \mathbf{w}_{t}^{(i)}\right\|^{2} / \chi^{(0)} \sim \mathcal{E}(1)$ is independent of $\chi^{(0)}$. Further details are available in [15].

\section{System-LEVEL ANALysis}

In SWIPT cellular networks, the trade-off between information rate and harvested power is quantified in terms of the J-CCDF of $\mathcal{R}$ and $\mathcal{Q}$ defined in (7). The J-CCDF is [11]:

$$
F_{c}\left(\mathcal{R}_{*}, \mathcal{Q}_{*}\right)=\operatorname{Pr}\left\{\mathcal{R} \geq \mathcal{R}_{*}, \mathcal{Q} \geq \mathcal{Q}_{*}\right\}
$$

where $\mathcal{R}_{*}$ and $\mathcal{Q}_{*}$ are the minimum data rate and harvested power needed for the LED being able to perform its tasks. 


$$
\begin{aligned}
\Phi_{\mathcal{I}}\left(\omega \mid L^{(0)} ; s\right) & =\exp \left(\lambda \pi q_{s}^{[D, \infty]} \max \left\{D^{2},\left(L^{(0)} / \kappa_{0}\right)^{2 / \beta_{s}}\right\}\left(1-\Upsilon_{s}\left(\omega, \max \left\{\kappa_{0} D^{\beta_{s}}, L^{(0)}\right\}\right)\right)\right) \\
& \times \exp \left(\pi \lambda q_{s}^{[0, D]}\left[\left(L^{(0)} / \kappa_{0}\right)^{2 / \beta_{s}}\left(1-\Upsilon_{s}\left(\omega, L^{(0)}\right)\right)-D^{2}\left(1-\Upsilon_{s}\left(\omega, \kappa_{0} D^{\beta_{s}}\right)\right)\right] \overline{\mathcal{H}}\left(L^{(0)}-\kappa_{0} D^{\beta_{s}}\right)\right)
\end{aligned}
$$

$$
\begin{aligned}
& \mathcal{J}_{a}^{(1)}\left(m_{a}, \theta_{a}\right)=\int_{0}^{+\infty} \int_{0}^{+\infty} \frac{1}{\pi \omega} \operatorname{Im}\left\{\exp \left(-j \omega \frac{q_{*}}{P \mathcal{G}_{M}}\right)\left(\frac{1}{\theta_{a}}-\frac{j \omega}{y}\right)^{-m_{a}} \Gamma\left(m_{a}, \frac{\mathcal{T}_{*}}{P \mathcal{G}_{M}}\left(\frac{y}{\theta_{a}}-j \omega\right)\right) \Phi_{\mathcal{I}}\left(\frac{\omega}{\mathcal{G}_{M}} \mid y\right)\right\} f_{L^{(0)}}(y) d \omega d y \\
& \mathcal{J}_{a}^{(2)}\left(m_{a}, \theta_{a}\right)=\int_{0}^{+\infty} \int_{0}^{+\infty} \frac{1}{\pi \omega} \operatorname{Im}\left\{\exp \left(j \omega \frac{\sigma_{*}^{2}}{P \mathcal{G}_{M}}\right)\left(\frac{1}{\theta_{a}}+\frac{j \omega r_{*}}{y}\right)^{-m_{a}} \Gamma\left(m_{a}, \frac{\mathcal{T}_{*}}{P \mathcal{G}_{M}}\left(\frac{y}{\theta_{a}}+j \omega r_{*}\right)\right) \Phi_{\mathcal{I}}\left(\frac{\omega}{\mathcal{G}_{M}} \mid y\right)\right\} f_{L^{(0)}}(y) d \omega d y
\end{aligned}
$$

\section{A. Exact Mathematical Framework}

Proposition 1: The J-CCDF in (9) can be formulated as:

$$
F_{c}\left(\mathcal{R}_{*}, \mathcal{Q}_{*}\right)=\mathcal{K}_{p, q} \sum_{v=1}^{q} \sum_{u=p-q}^{(p+q-2 v) v} c_{v, u}\left(\mathcal{J}_{v, u}^{(1)}-\mathcal{J}_{v, u}^{(2)}\right)
$$

where $r_{*}=\left(2^{\mathcal{R}_{*} / B_{w}}-1\right)^{-1}, \sigma_{*}^{2}=\sigma_{N}^{2}+\sigma_{\mathrm{ID}}^{2}(1-\rho)^{-1}, q_{*}=$ $\mathcal{Q}_{*}(\rho \zeta)^{-1}, \mathcal{T}_{*}=\left(q_{*}+\sigma_{*}^{2}\right) /\left(r_{*}+1\right), \mathcal{J}_{v, u}^{(1)}$ and $\mathcal{J}_{v, u}^{(2)}$ are in (11) (see previous page), $f_{L^{(0)}}(\cdot)$ is the PDF of $L^{(0)}$ :

$$
f_{L^{(0)}}(x)=\widehat{\Lambda}([0, x)) \exp (-\Lambda([0, x))),
$$

$\Lambda([0, x))=\Lambda_{\mathrm{LOS}}([0, x))+\Lambda_{\mathrm{NLOS}}([0, x)), \widehat{\Lambda}([0, x))=$ $\widehat{\Lambda}_{\mathrm{LOS}}([0, x))+\widehat{\Lambda}_{\mathrm{NLOS}}([0, x))$, and $\Lambda_{s}([\cdot, \cdot)), \widehat{\Lambda}_{s}([\cdot, \cdot))$ are:

$$
\begin{aligned}
& \Lambda_{s}([0, x))=\pi \lambda q_{s}^{[0, D]}\left(\frac{x}{\kappa_{0}}\right)^{\frac{2}{\beta_{s}}} \overline{\mathcal{H}}\left(x-\kappa_{0} D^{\beta_{s}}\right) \\
& +\pi \lambda\left(\left(\frac{x}{\kappa_{0}}\right)^{\frac{2}{\beta_{s}}} q_{s}^{[D, \infty]}+D^{2}\left(q_{s}^{[0, D]}-q_{s}^{[D, \infty]}\right)\right) \mathcal{H}\left(x-\kappa_{0} D^{\beta_{s}}\right) \\
& \widehat{\Lambda}_{s}([0, x))=\left(2 \pi \lambda / \beta_{s}\right) q_{s}^{[0, D]} \kappa_{0}^{-2 / \beta_{s}} x^{\left(2 / \beta_{s}-1\right)} \overline{\mathcal{H}}\left(x-\kappa_{0} D^{\beta_{s}}\right) \\
& +\left(2 \pi \lambda / \beta_{s}\right) q_{s}^{[D, \infty]} \kappa_{0}^{-2 / \beta_{s}} x^{\left(2 / \beta_{s}-1\right)} \mathcal{H}\left(x-\kappa_{0} D^{\beta_{s}}\right)
\end{aligned}
$$

as well as $\Phi_{\mathcal{I}}\left(\cdot \mid L^{(0)}\right)$ is the CF of $\mathcal{I}$ conditioned on $L^{(0)}$ :

$$
\Phi_{\mathcal{I}}\left(\omega \mid L^{(0)}\right)=\Phi_{\mathcal{I}}\left(\omega \mid L^{(0)} ; \operatorname{LOS}\right) \Phi_{\mathcal{I}}\left(\omega \mid L^{(0)} ; \mathrm{NLOS}\right)
$$

where $\Phi_{\mathcal{I}}\left(\cdot \mid L^{(0)} ; s\right)$ is in (15) at the top of this page and $\Upsilon_{s}(\omega, Z)=\left(\omega_{M} / 2 \pi\right){ }_{2} F_{1}\left(1,-2 / \beta_{s}, 1-2 / \beta_{s}, j \omega \mathcal{G}_{M} / Z\right)+$ $\left(1-\omega_{M} / 2 \pi\right){ }_{2} F_{1}\left(1,-2 / \beta_{s}, 1-2 / \beta_{s}, j \omega \mathcal{G}_{m} / Z\right)$.

Proof: It follows by using an approach similar to [11].

The J-CCDF in (10) is exact but the number of addends of the summations in (10) increases with $N_{t}$ or $N_{r}$.

\section{B. Approximated Mathematical Framework}

To increase the computational efficiency of (10) and the insight that can be gained from it, we approximate the PDF of $\chi^{(0)}$ in (5) with that of a gamma RV $\tilde{\chi}^{(0)}$, i.e., $\chi^{(0)} \approx \tilde{\chi}^{(0)}$ :

$$
f_{\tilde{\chi}^{(0)}}(\xi)=\xi^{m_{a}-1} /\left(\Gamma\left(m_{a}\right) \theta_{a}^{m_{a}}\right) \exp \left(-\xi / \theta_{a}\right)
$$

where $\mu_{\tilde{\chi}^{(0)}}=\mathbb{E}\left\{\tilde{\chi}^{(0)}\right\}=m_{a} \theta_{a}$ and $\eta_{\tilde{\chi}^{(0)}}^{2}=\mathbb{E}\left\{\left(\tilde{\chi}^{(0)}\right)^{2}\right\}-$ $\left(\mathbb{E}\left\{\tilde{\chi}^{(0)}\right\}\right)^{2}=m_{a} \theta_{a}^{2}$ are the mean and the variance of $\tilde{\chi}^{(0)}$. The pair $\left(\mu_{\tilde{\chi}^{(0)}}, \eta_{\tilde{\chi}^{(0)}}^{2}\right)$ is obtained from the moment matching method, i.e., by solving the system of equations $\mathbb{E}\left\{\chi^{(0)}\right\}=$ $\mu_{\tilde{\chi}^{(0)}}$ and $\mathbb{E}\left\{\left(\chi^{(0)}\right)^{2}\right\}-\left(\mathbb{E}\left\{\chi^{(0)}\right\}\right)^{2}=\eta_{\tilde{\chi}^{(0)}}^{2}$, which yields:

$$
m_{a}=\frac{\left(\mathbb{E}\left\{\chi^{(0)}\right\}\right)^{2}}{\mathbb{E}\left\{\left(\chi^{(0)}\right)^{2}\right\}-\left(\mathbb{E}\left\{\chi^{(0)}\right\}\right)^{2}} \quad \theta_{a}=\frac{\mathbb{E}\left\{\left(\chi^{(0)}\right)^{2}\right\}-\left(\mathbb{E}\left\{\chi^{(0)}\right\}\right)^{2}}{\mathbb{E}\left\{\chi^{(0)}\right\}}
$$

where $\mathbb{E}\left\{\left(\chi^{(0)}\right)^{\eta}\right\}=\mathcal{K}_{p, q} \sum_{v=1}^{q} \sum_{u=p-q}^{(p+q-2 v) v}\left(c_{v, u} / v^{u+\eta+1}\right)$ $\times \Gamma(u+\eta+1)$ is the $\eta$ th moment of $\chi^{(0)}$ obtained from (5).

Proposition 2: The J-CCDF in (9) can be approximated as:

$$
F_{c}\left(\mathcal{R}_{*}, \mathcal{Q}_{*}\right) \approx \frac{1}{\Gamma\left(m_{a}\right) \theta_{a}^{m_{a}}}\left(\mathcal{J}_{a}^{(1)}\left(m_{a}, \theta_{a}\right)-\mathcal{J}_{a}^{(2)}\left(m_{a}, \theta_{a}\right)\right)
$$

where $\mathcal{J}_{a}^{(1)}(\cdot, \cdot), \mathcal{J}_{a}^{(2)}(\cdot, \cdot)$ are in (19) at the top of this page.

Proof: It is similar to the proof of Proposition 1.

Compared with the approach in [12], Proposition 2 is more accurate, as it is applicable for any $m_{a}$ and not just for $m_{a} \gg$ 1. Thanks to the single-ball blockage model in (1), it is more tractable as well [13]. As a result, it is useful for analyzing both centimeter- and millimeter-wave cellular networks.

\section{Asymptotic (Large-Scale) Mathematical Framework}

Proposition 3: In the large-scale MIMO regime, i.e., $N_{t} \gg$ 1 or $N_{r} \gg 1$, the J-CCDF in (9) is asymptotically equal to:

$$
\begin{aligned}
F_{c}\left(\mathcal{R}_{*}, \mathcal{Q}_{*}\right) \rightarrow & \int_{0}^{+\infty} \int_{0}^{\tilde{y}_{\mathrm{M}}}(\pi \omega)^{-1} f_{L^{(0)}}(y) \\
& \times \operatorname{Im}\left\{\mathcal{J}_{\infty}\left(\mu_{\tilde{\chi}^{(0)}} ; \omega, y\right) \Phi_{\mathcal{I}}\left(\omega / \mathcal{G}_{M} \mid y\right)\right\} d \omega d y
\end{aligned}
$$

where $\tilde{y}_{\mathrm{M}}=\mu_{\tilde{\chi}^{(0)}} P \mathcal{G}_{M} / \mathcal{T}_{*}$ and $\mathcal{J}_{\infty}(\cdot ; \cdot, \cdot)$ is defined as:

$$
\begin{aligned}
\mathcal{J}_{\infty}\left(\mu_{\tilde{\chi}^{(0)}} ; \omega, y\right) & =\exp \left(-j \omega\left(q_{*} / P \mathcal{G}_{M}-\mu_{\tilde{\chi}^{(0)}} / y\right)\right) \\
& -\exp \left(-j \omega\left(-\sigma_{*}^{2} / P \mathcal{G}_{M}+r_{*} \mu_{\tilde{\chi}^{(0)}} / y\right)\right)
\end{aligned}
$$

Proof: It follows from (18), setting $\theta_{a}=\mu_{\tilde{\chi}^{(0)}} / m_{a}$, noting, from (17), that $m_{a} \rightarrow \infty$ if $N_{t} \rightarrow \infty$ or $N_{r} \rightarrow \infty$, and:

$$
\begin{aligned}
& \lim _{m_{a} \rightarrow+\infty} \Gamma\left(m_{a}, z\right) / \Gamma\left(m_{a}\right)=\mathbf{1}\left(\operatorname{Re}\{z\} \leq m_{a}\right) \\
& \lim _{m_{a} \rightarrow+\infty}\left(1+z / m_{a}\right)^{-m_{a}}=\exp (-z)
\end{aligned}
$$

which hold for any $z \in \mathbb{C}$ whose real part is non-negative. $\square$

From the Gil-Pelaez theorem [16], (20) can be written as:

$$
\begin{array}{r}
F_{c}\left(\mathcal{R}_{*}, \mathcal{Q}_{*}\right) \rightarrow \mathbb{E}_{L^{(0)}}\left\{\operatorname{Pr}\left\{\mathcal{I} \geq\left(q_{*} / P \mathcal{G}_{M}-\mu_{\tilde{\chi}^{(0)}} / L^{(0)}\right) \mid L^{(0)}\right\}\right\} \\
-\mathbb{E}_{L^{(0)}}\left\{\operatorname{Pr}\left\{\mathcal{I} \geq\left(-\sigma_{*}^{2} / P \mathcal{G}_{M}+r_{*} \mu_{\tilde{\chi}^{(0)}} / L^{(0)}\right) \mid L^{(0)}\right\}\right\}
\end{array}
$$

for $L^{(0)} \leq \mu_{\tilde{\chi}^{(0)}} P \mathcal{G}_{M} / \mathcal{T}_{*}$ and $F_{c}\left(\mathcal{R}_{*}, \mathcal{Q}_{*}\right) \rightarrow 0$ otherwise.

As for the impact of $N_{t}$ and $N_{r}$, the following conclusions can be drawn, in the large-scale antenna regime, from (23).

- The J-CCDF depends only on the average strength of the intended link: $\mu_{\tilde{\chi}^{(0)}}=\mathbb{E}\left\{\tilde{\chi}^{(0)}\right\}=\mathbb{E}\left\{\chi^{(0)}\right\}=\mu_{\chi^{(0)}}$.

- The J-CCDF increases as $\mu_{\tilde{\chi}^{(0)}}$ increases, since, by definition, the first and the second addend of (23) increases and decreases, respectively, as a function of $\mu_{\tilde{\chi}^{(0)}}$.

From its definition in (17), $\mu_{\chi^{(0)}}$ increases as either $N_{t}$ or $N_{r}$ increases. We conclude that, for any $\mathcal{R}_{*}$ and $\mathcal{Q}_{*}$, 


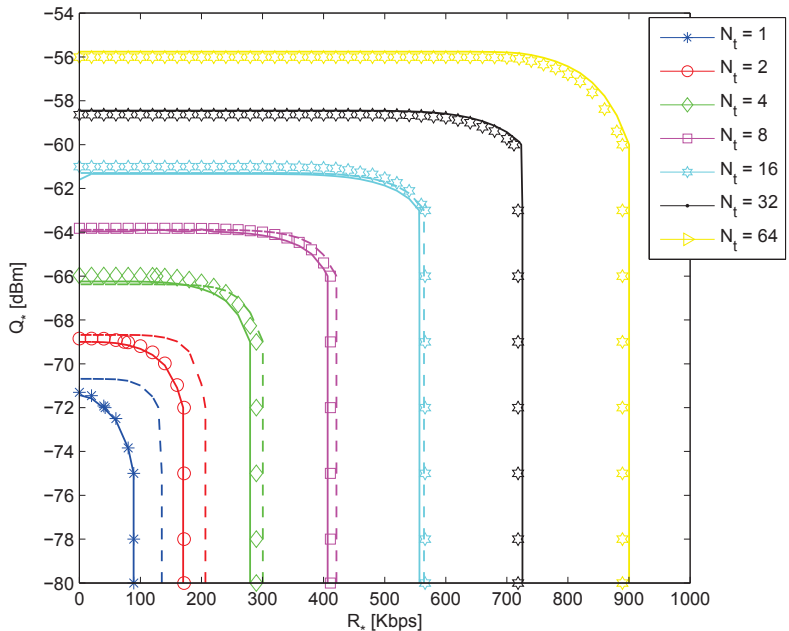

Fig. 1. Contour lines of J-CCDF vs. $N_{t}\left(\rho=0.5, R_{\text {cell }}=83.4122 \mathrm{~m}\right.$ [14], $\mathcal{G}_{M}=1, \mathcal{G}_{m}=1, \omega_{M}=360$ degrees (omnidirectional antennas) [13]) They show the pairs $\left(\mathcal{R}_{*}, \mathcal{Q}_{*}\right)$ so that $F_{c}\left(\mathcal{R}_{*}, \mathcal{Q}_{*}\right)=0.75$. Solid lines show exact and approximated frameworks in (10) and (18). Dashed lines show the asymptotic framework in (20). Markers show Monte Carlo simulations.
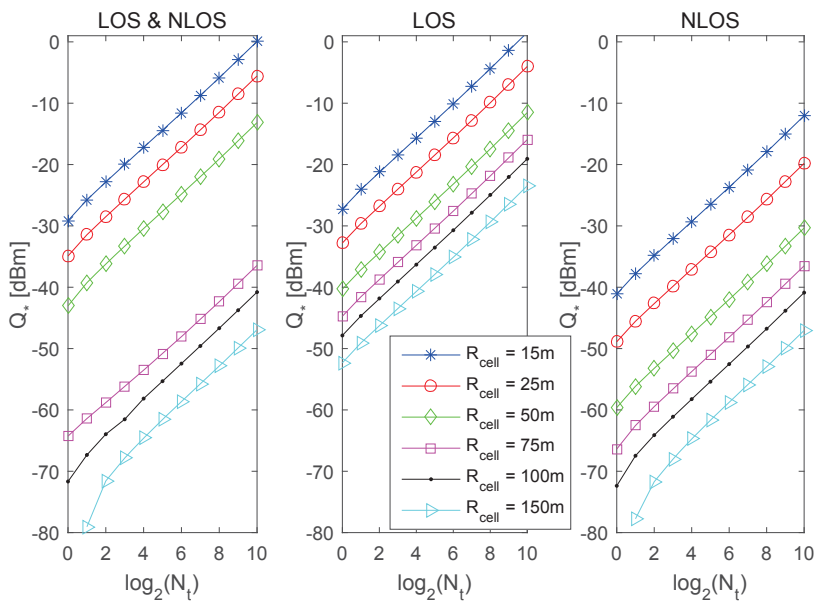

Fig. 2. Harvested power $\mathcal{Q}_{*}$ vs. $N_{t}$ and $R_{\text {cell }}$ so that $F_{c}\left(\mathcal{R}_{*}, \mathcal{Q}_{*}\right)=0.90$ and $\mathcal{R}_{*}=100 \mathrm{kbits} / \mathrm{sec} . \mathcal{G}_{M}=32.4195, \mathcal{G}_{m}=0.4363, \omega_{M}=12$ degrees (directive antennas) [13]. The curves are obtained by using (18) and each point corresponds to the optimal $\rho$ that maximizes the JCCDF.

$F_{c}\left(\mathcal{R}_{*}, \mathcal{Q}_{*}\right)$ can be arbitrary close to one if either $N_{t}$ or $N_{r}$ are sufficiently large. Due to the limited size of the LEDs, this implies that the BSs need to be equipped with many antennas.

\section{Numerical AND Simulation RESUlts}

Considered setup: $\nu=c_{0} / f_{c}$, where $c_{0}$ is the speed of light in $\mathrm{m} / \mathrm{sec}$ and $f_{c}=2.1 \mathrm{GHz}$ is the carrier frequency; $\sigma_{\mathrm{ID}}^{2}=-70 \mathrm{dBm} ; \sigma_{N}^{2}=-174+10 \log _{10}\left(B_{w}\right)+\mathcal{F}_{N} \mathrm{dBm}$, where $B_{w}=200 \mathrm{KHz}$ and $\mathcal{F}_{N}=10 \mathrm{~dB}$ is the noise figure; $P=30 \mathrm{dBm} ; \zeta=0.8 ; \lambda=1 /\left(\pi R_{\text {cell }}^{2}\right)$ where $R_{\text {cell }}$ is the average cell radius. The channel model is [14]: $D=109.8517$ $\mathrm{m} ; q_{\mathrm{LOS}}^{[0, D]}=0.7195 ; q_{\mathrm{LOS}}^{[D, \infty]}=0.0002 ; \beta_{\mathrm{LOS}}=2.5 ; \beta_{\mathrm{NLOS}}=$ 3.5. $N_{r}=2$ is assumed, which may be applicable to smartwatches, as the average circumference of a human wrist is 14-20 cm. Simulations (details can be found in [14]) and frameworks are obtained with Matlab and Mathematica.
In Fig. 1, we validate the accuracy of the mathematical frameworks as a function of $N_{t}$. The exact and approximated frameworks in (10) and (18) are indistinguishable from each other. The asymptotic framework in (20) becomes tighter as $N_{t}$ increases. In the considered setup, it is accurate enough for $N_{t}=4$. In Fig. 2, we perform a feasibility study of SWIPT cellular networks. The curves are obtained from (18) by computing $\mathcal{Q}_{*}$ that corresponds to the optimal $F_{c}(\cdot, \cdot)$ as a function of $\rho$ and such that $\mathcal{R}_{*}=100 \mathrm{kbits} / \mathrm{sec}$ and $F_{c}\left(\mathcal{R}_{*}, \mathcal{Q}_{*}\right)=0.90$. The existence of an optimal $\rho$ originates from the fact that $\mathcal{Q}$ increases and $\mathcal{R}$ decreases with $\rho$. Fig. 2 shows that different results are obtained if LOS and NLOS links are neglected. This justifies the adoption of the considered blockage model. Fig. 2 shows, in addition, that network densification and large-scale MIMO are both essential for enabling SWIPT cellular networks harvest an amount of power of the order of a milliwatt, while still guaranteeing a sufficient rate for LEDs applications. It is worth nothing that the performance trends shown in Figs. 1 and 2 assume that the input-output response of the EH receiver is linear with the input power. The analysis of non-linear EH models [17] may, on the other hand, result in different performance trends.

\section{REFERENCES}

[1] I. Krikidis et al., "Simultaneous wireless information and power transfer in modern communication systems”, IEEE Commun. Mag., vol. 52, no. 11, pp. 104-110, Nov. 2014.

[2] http://www.cnet.com/news/ring-ring-its-your-watch-calling-att-bets.

[3] M. Di Renzo et al., "Average rate of downlink heterogeneous cellular networks over generalized fading channels - A stochastic geometry approach”, IEEE Trans. Commun., vol. 61, no. 7, pp. 3050-3071, July 2013.

[4] G. Zheng et al., "Rethinking the role of interference in wireless networks”, IEEE Commun. Mag., vol. 52, no. 11, pp. 152-158, Nov. 2014.

[5] Z. Ding et al., "Application of smart antenna technologies in simultaneous wireless information and power transfer", IEEE Commun. Mag., vol. 53, no. 4, pp. 86-93, Apr. 2015.

[6] I. Krikidis, "Simultaneous information and energy transfer in large-scale networks with/without relaying”, IEEE Trans. Commun., vol. 62, no. 3, pp. 900-912, Mar. 2014.

[7] Z. Ding et al., "Wireless information and power transfer in cooperative networks with spatially random relays”, IEEE Trans. Wireless Commun., vol. 13,, no. 10, pp. 4440-4453, Aug. 2014.

[8] J. Guo et al., "Outage probability of ad hoc networks with wireless information and power transfer", IEEE Wireless Commun. Lett., vol. 4, no. 8, pp. 409-412, Aug. 2015.

[9] I. Flint et al., "Performance analysis of ambient RF energy harvesting with repulsive point process modeling”, IEEE Trans. Wireless Commun., vol. 14,, no. 10, pp. 5402-5416, Oct. 2015.

[10] X. Zhou et al., "Performance of maximum ratio transmission in ad hoc networks with SWIPT", IEEE Wireless Commun. Lett., vol. 4, no. 10, pp. 529-532, Oct. 2015.

[11] W. Lu et al., "On stochastic geometry analysis and optimization of wireless-powered cellular networks”, IEEE GLOBECOM, Dec. 2015.

[12] T. A. Khan et al., "Millimeter wave energy harvesting", submitted. http://arxiv.org/pdf/1509.01653v2.pdf.

[13] M. Di Renzo et al., "The intensity matching approach: A tractable stochastic geometry approximation to system-level analysis of cellular networks", IEEE Trans. Wireless Commun., to appear.

[14] W. Lu et al., "Stochastic geometry modeling of cellular networks: Analysis, simulation, experimental validation”, ACM MSWiM, Nov. 2015.

[15] A. Maaref and S. Aissa, "Closed form expressions for the outage and ergodic shannon capacity of MIMO MRC systems”, IEEE Trans. Commun., vol. 53, no. 7, pp. 1092-1095, July 2005.

[16] J. Gil-Pelaez, "Note on the inversion theorem”, Biometrika, Dec. 1951.

[17] E. Boshkovska et al., "Practical non-linear energy harvesting model and resource allocation for SWIPT systems”, IEEE Commun. Lett., vol. 19, no. 12, pp. 2082-2085, Dec. 2015. 